\title{
Ethnic differences on cardiovascular disease risk and quality of life in Selangor
}

\author{
Lee, H.S., Aman, A., Kamaruddin, K.S., Ali, A. and *Yusof, H.M. \\ Faculty of Fisheries and Food Science, Universiti Malaysia Terengganu, 21030 Kuala Nerus, Terengganu, \\ Malaysia
}

\begin{abstract}
Article history:
Received: 26 February 2020

Received in revised form: 29

March 2020

Accepted: 1 April 2020

Available Online: 19 April 2020
\end{abstract}

\section{Keywords:}

Cardiovascular disease risk, Quality of life,

Multiethnic,

Retrospective study

DOI:

https://doi.org/10.26656/fr.2017.4(4).035

\begin{abstract}
Malaysia is currently experiencing an epidemiologic and demographic transition. Rapid urbanization and vast changes in lifestyles among the population has led to widespread cardiovascular disease (CVD) outcomes. Also, until now, the most prevalent cardiovascular risk factor has remained unknown among the Malaysian population. This study aimed to identify the most significant cardiovascular risk factor among multiethnic adults in Dengkil, Selangor. A case-control retrospective study comparing past exposure (retrospective) between the case (total cholesterol (TC) $\geq 5.18 \mathrm{mmol} / \mathrm{L}$ ) and control groups $(\mathrm{TC}<5.18 \mathrm{mmol} / \mathrm{L})$ on CVD risk via guided questionnaire comprised of International Physical Activity Questionnaire (IPAQ), Food Frequency Questionnaire (FFQ), Depression, Anxiety and Stress Scales (DASS)-21 and SF-12 was carried out among 180 respondents aged 20 to 56 years in Dengkil, Selangor. In the present study, purposive and consecutive sampling were both applied to recruit respondents and also considering ethnic as a factor; thus, quota sampling was also conducted. The data were analyzed using Mann-Whitney, Kruskal-Wallis, Chi-Square, Independent Samples t-test and multiple logistic regression tests. The findings indicated there were no significant differences $(p>0.05)$ regarding all the risk factors between the case and control groups except for personal medical history $(\mathrm{p}<0.05)$. Chinese possessed the highest number of significant risk and protective factors. Lastly, the quality of life of Malay controls was higher than that of cases in terms of Physical Component Summary (PCS). Generally, all the risk factors were independent in terms of total cholesterol status, except for personal medical history. The significance of risk factors and protective factors varies according to ethnicity.
\end{abstract}

\section{Introduction}

Cardiovascular disease (CVD) is a series of malfunctions in the circulatory system, especially in the heart and blood vessels. This includes coronary heart disease and ischemic heart disease (IHD), cerebrovascular disease, and other heart complications. The most common CVD are IHD and cerebrovascular disease (Roth et al., 2015).

The severity of CVD among Malaysians is supported through data collected from the Department of Statistics Malaysia (2017), indicating the primary cause at 20.1\% of deaths among Malaysians was CVD, ranking it as the number one 'killer' among Malaysians. Obviously, there is an increasing trend in the prevalence of cardiovascular risk factors - hypertension, hypercholesterolemia, diabetes, overweight/obesity and smoking (Kumar,
2014). It has also shown that Malaysians who develop heart disease are younger from year to year and becoming more serious in recent years as compared to neighboring countries such as Singapore and Thailand (Aniza et al., 2016). Thus, necessary prevention strategies such as population-based and high-risk individual-based intervention are crucial to lowering the prevalence of CVD in Malaysia (Pandey et al., 2013).

Basically, the common causes of CVD can be divided into two major categories, namely modifiable and non-modifiable risk factors. Examples of modifiable risk factors are hypertension, stroke and diabetes mellitus, whereas examples of non-modifiable risk factors are age, ethnic, socioeconomic status, family history and sex (Aniza et al., 2016; André et al., 2017). In addition, another important predictor for CVD is health-related quality of life (HRQoL). HRQoL 
measures the implications of and influences on quality of life caused by the symptoms and severity of diseases. Impaired HRQoL is closely associated with an increased risk of mortality and hospitalization due to CVD (Thompson et al., 2016).

CVD can occur everywhere, every time, and to everybody. Unfortunately, until now, the lack of comprehensive case-control studies to investigate the most significant CVD risk factors among multiethnic adults in Malaysia. Previous studies mainly focus on certain highlighted risk factors, for instance, hypertension, without taking other risk factors such as diabetes, stroke, dietary pattern into considerations (Akter et al., 2010; Thon et al., 2012; Juhan et al., 2018). The most recent case-control retrospective study was done in Terengganu among Malay adults only (Erl et al., 2019). To the best of author's knowledge, there are limited findings on the most significant CVD risk among multiethnic adults. This study is useful to identify the highest prevalence of CVD risk by investigating various CVD risk factors according to ethnicity. These findings are especially important for the implementation of required interventions which are essential in reducing the prevalence of CVD now and in the future.

The implementation of this study design, which is a retrospective case-control study, is due to several reasons. Firstly, it is quick and inexpensive. Furthermore, it requires fewer subjects and is best suited to investigate outcomes with a long latency period (Song and Chung, 2010). As such, this research was conducted in Dengkil, a small town located in between the rural and urban area at Sepang district in Selangor. According to the Department of Statistics Malaysia (2015), the ethnic groups of adults in Dengkil are well-distributed, with Malays $(71.3 \%)$, Chinese $(15.7 \%)$ and Indians (13\%), which is approximately same as the ethnic distribution in Malaysia. Additionally, the proportion of adult in Dengkil $(60.5 \%)$ is similar to the adult proportion in Malaysia (69.7\%). Thus, the geography and demography of Dengkil, Selangor provided motivation for this study to be conducted. Necessary interventions regarding CVD can be generated through this study.

The present study aims to determine the most significant CVD risk factors among multiethnic. Besides, it aims to compare the past exposure (retrospective) including the quality of life between case and control groups on CVD risk, in addition, to identify the ethnic with highest CVD risk in Selangor. On the other hand, this study aims to determine the most prevalent CVD risk and protective factor among multiethnic populations.

\section{Materials and methods}

\subsection{Research design and subjects}

A case-control retrospective study was carried out in Dengkil, Selangor. The respondents were selected via purposive sampling, from June to August 2018. Kelsey et al. (1996) were used for sample size calculation for the confidence level of $95 \%$. The study was done at hospitals, clinics and restaurants to represent adult the population in Dengkil. Thus, a total of 180 adults comprised of 60 Malays, 60 Chinese and 60 Indians were recruited through consecutive and quota sampling considering ethnic as a factor as shown in Figure 1. Consecutive sampling and quota sampling, which are non-probability samplings, were used to select specific locations and respondents for the present study. Consecutive sampling was used in selecting the subjects who meet the criteria of inclusion from an accessible population over a specific time interval (Mathieson, 2014). The inclusion criteria for this study were those who possessed known values for serum lipid profiles as well as willing to answer the questionnaires honestly. However, there were also some criteria of exclusion such as pregnancy and hemophilia. Quota sampling was used when a quota was then set for each sub-group which directs the sample to be approached on the basis of set quota (Alvi, 2016). For this study, the equal numbers of subjects between cases group and controls group were obtained by using a quota sampling with 1:1 ratio (90 controls and 90 cases) in the sampling location. The control and case groups were subdivided into three smaller groups, namely Malays, Chinese and Indians, respectively.

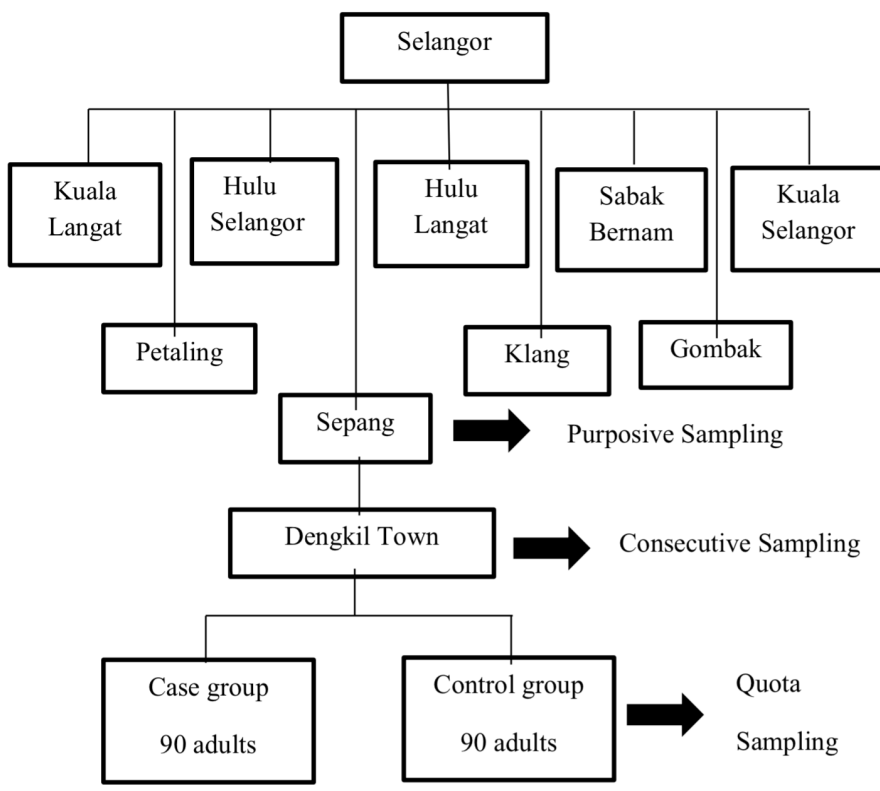

Figure 1. Sampling framework

\subsection{Ethical approval}

The present study was approved by National Medical Research Register (NMRR) of National Institutes of 
Health (NIH) under the Ministry of Health with registration number NMRR-18-906-41488 (IIR). Furthermore, consent forms were signed and collected from all study participants.

\subsection{Research instrument}

A guided questionnaire was used as the research instrument in the present study. The questionnaire is comprised of six sections: socio-demographic information, medical and health status, lifestyle behaviors, dietary assessment assessed by Food Frequency Questionnaire (FFQ), psychosocial status assessment using the Depression, Anxiety and Stress Scales-21 (DASS-21). HRQoL assessment using the 12item short-form health survey (SF-12).

\subsection{Physical Activity (IPAQ)}

The scoring guidelines for short form IPAQ were based on the latest existing guidelines (IPAQ Analysis, 2005). Classification of the respondents into three categories was performed once the results of total physical activity Metabolic Equivalent Task (MET)minutes/week were obtained. The low, sedentary or inactive group of respondents engage in less than 600 MET-minutes/week of exercise, while the minimally inactive group (moderate physical activity level) engages in 600-3000 MET-minutes/week of exercise, and the physically active group (high physical activity level) engages in higher than 3000 MET-minutes/week of exercise (IPAQ Analysis, 2005).

\subsection{Dietary intake (FFQ)}

The FFQ used in this study included fifteen food items (Chee et al., 1996). Lower scores mean lower intake for a particular food. The ranges were classified into four categories. Scores of less than 20.0 means extremely less consumed foods whereas scores of 20.059.9 indicate less consumed foods. On the other hand, scores of 60.0-79.9 indicate moderately consumed foods whereas scores if 80.0-100.0 indicates highly consumed foods.

\subsection{Psychosocial assessment (DASS 21)}

The scoring of DASS-21 was based on an established scoring manual by Lovibond and Lovibond (1995). The final scores were calculated by doubling the total scores for the relevant items for depression, anxiety and stress. Higher scores indicate weaker psychosocial status. Good psychosocial status indicates subjects without either/ at least one of depression, anxiety and stress whereas poor psychosocial status indicate subjects with either/ at least one of depression, anxiety and stress.

\subsection{Quality of life (SF-12)}

HRQoL was assessed by using the 12-item shortform health survey (SF-12). Short Form (SF)-12 Health Survey Standard Scoring (2nd edition) was used to score the quality of life of respondents based on Physical Component Summary (PCS) and Mental Component Summary (MCS) (Ware et al., 1994; Gandek et al., 2004). The scores range from 0 (lowest health level) to 100 (highest health level).

\subsection{Risk and protective factors of $C V D$}

Socioeconomic status was analyzed from the education level and monthly household income. Low education level (primary and secondary education) and low household income (less than RM3,000) considered as the risk factors of CVD. On the other hand, protective factors of CVD were identified by high education (university and college level) and high monthly household income (more than RM6,500). In addition, risk factors of CVD were studied by personal and family medical history (with at least one of the disease such as hypertension, diabetes and heart problems), low physical activity, smoking, alcohol drinking and poor psychosocial status (with at least one among depression, anxiety and stress). Absence of personal and family medical history, high physical activity, no smoking, absence of alcohol drinking and good psychosocial status were the other aspects used to measure the protective factors of CVD. Odds Ratio (OR) was then used to compare the CVD risk and protective factors between case and control groups according to ethnicity.

\subsection{Data analysis}

The data was analyzed using SPSS version 20. Normality tests (Kolmogorov-Smirnov and Shapiro Wilk tests) were used to examine the distribution of the data of the variables. Descriptive tests were conducted to analyses the socio-demographic profiles and quality of life of respondents.

In this study, Chi-square was used to test the dependent variables which were categorized as categorical data. An independent-samples $t$-test was applied for the normally distributed data whereas MannWhitney $U$ test was performed for the non-normally distributed data. OR is defined as a relative measure of effect which permits the comparison of the group with specific outcomes relative to the placebo group. In this study, OR was used to examine the relative risk for CVD from a specific risk factor between case and control groups (Schmidt and Kohlmann, 2008). An unmatched case-control method was used in this study. The calculation of $p$-value was followed by the $95 \%$ confidence interval (CI). (Greenland et al., 2016). 


\section{Results}

\subsection{Socio-demographic characteristics of respondents}

Socio-demographic characteristics of the respondents involved in this study are shown in Table 1. The mean age for the control group is $36.2 \pm 9.5$, with 40 (44.4) males and 50 (55.6) females. On the other hand, the mean age for the case group is $39.7 \pm 9.1$ with 45 (50) males and 45 (50) females. The study found that the mean body weight for the controls was $61.0 \mathrm{~kg}$ with a mean of BMI of $22.2 \mathrm{~kg} / \mathrm{m}^{2}$, which is still classified as normal BMI. The BMI values for the three ethnic groups are approximately the same, and still within normal BMI. Thus, the mean body weight for the case investigated is $61.4 \mathrm{~kg}$, with a median BMI of $22.3 \mathrm{~kg} / \mathrm{m}^{2}$.

\subsection{Association of CVD risk factors between controls and cases of different ethnic groups}

Table 2 shows the comparison of CVD risk between control and case multiethnic groups. CVD risk factors were significantly different according to ethnicity. In total, all the risk factors showed no significant difference $(p>0.05)$ except for personal medical history $(p<$
0.001). Malay and Indian respondents showed significant differences between groups $(p<0.001)$ in terms of personal medical history such as hypertension, diabetes mellitus, hypercholesterolemia, and stroke. On the other hand, Chinese respondents showed significant differences between the case-control groups for personal medical history $(\mathrm{p}<0.001)$ in addition to smoking $(\mathrm{p}=$ 0.002 ).

For all the ethnicities under investigation, no significant difference $(p>0.05)$ between controls and cases in terms of depression, anxiety and stress were revealed. However, when the comparison was done between ethnic groups, there were significant differences $(p<0.05)$ among the ethnicities of both groups.

The scores for psychosocial status are the highest in Malays, followed by Indians and Chinese. On the other hand, the scores for depression, anxiety and stress of cases are higher than controls, but the difference is not significant $(\mathrm{p}>0.05)$.

Table 1. Socio-demographic characteristics of respondents

\begin{tabular}{|c|c|c|c|c|c|c|c|c|}
\hline \multirow[b]{2}{*}{ Category, n (\%) } & \multicolumn{4}{|c|}{ Control $(n=90)$} & \multicolumn{4}{|c|}{ Case $(n=90)$} \\
\hline & $\begin{array}{l}\text { Malay } \\
(n=30)\end{array}$ & $\begin{array}{l}\text { Chinese } \\
(\mathrm{n}=30)\end{array}$ & $\begin{array}{l}\text { Indian } \\
(\mathrm{n}=30)\end{array}$ & Total & $\begin{array}{l}\text { Malay } \\
(n=30)\end{array}$ & $\begin{array}{l}\text { Chinese } \\
(\mathrm{n}=30)\end{array}$ & $\begin{array}{l}\text { Indian } \\
(\mathrm{n}=30)\end{array}$ & Total \\
\hline \multicolumn{9}{|l|}{ Gender } \\
\hline Male & $16(46.7)$ & $12(40.0)$ & $12(40.0)$ & $40(44.4)$ & $13(43.3)$ & $18(60.0)$ & $14(46.7)$ & $45(50.0)$ \\
\hline Female & $14(46.7)$ & $18(60.0)$ & $18(60.0)$ & $50(55.6)$ & $17(56.7)$ & $12(40.0)$ & $16(53.3)$ & $45(50.0)$ \\
\hline Age (year) & $36.9 \pm 9.5$ & $33.9 \pm 9.2$ & $36.2 \pm 9.5$ & $36.2 \pm 9.5$ & $39.2 \pm 9.2$ & $39.5 \pm 9.7$ & $40.5 \pm 8.7$ & $39.7 \pm 9.1$ \\
\hline Weight (kg) & $62.7 \pm 10.2$ & $59.6 \pm 11.0$ & $60.8 \pm 9.1$ & $61.0 \pm 10.1$ & $61.4 \pm 9.9$ & $61.7 \pm 10.5$ & $61.1 \pm 8.8$ & $61.4 \pm 9.7$ \\
\hline Height (cm) & $167.0 \pm 10.9$ & $165.4 \pm 8.2$ & $164.4 \pm 5.8$ & $165.5 \pm 8.6$ & $163.2 \pm 9.5$ & $166.5 \pm 9.4$ & $164.6 \pm 8.9$ & $164.5 \pm 8.9$ \\
\hline $\operatorname{BMI}\left(\mathrm{kg} / \mathrm{m}^{2}\right)$ & $22.2 \pm 2.7$ & $21.8 \pm 3.2$ & $22.5 \pm 3.0$ & $22.2 \pm 3.0$ & $23.2 \pm 5.0$ & $22.3(5.0)$ & $21.7(5.9)$ & $22.3(5.4)$ \\
\hline \multicolumn{9}{|l|}{ BMI group } \\
\hline Underweight & $3(10.0)$ & $3(10.0)$ & $2(6.7)$ & $8(8.9)$ & $2(6.7)$ & $3(10.0)$ & $3(10.0)$ & $8(8.9)$ \\
\hline Normal & $24(80.0)$ & $22(73.3)$ & $23(76.7)$ & $60(76.7)$ & $18(60.0)$ & $21(70.0)$ & $19(63.3)$ & $58(64.4)$ \\
\hline Overweight & $3(10.0)$ & $5(16.7)$ & $5(16.7)$ & $13(14.4)$ & $9(30.0)$ & $5(16.7)$ & $6(20.0)$ & $20(22.2)$ \\
\hline Obesity & $0(0.0)$ & $0(0.0)$ & $0(0.0)$ & $0(0.0)$ & $1(3.3)$ & $1(3.3)$ & $2(6.7)$ & $4(4.4)$ \\
\hline \multicolumn{9}{|c|}{ Highest education level } \\
\hline Primary & $3(10.0)$ & $1(3.3)$ & $4(13.3)$ & $8(8.9)$ & $6(20.0)$ & $1(3.3)$ & $6(20.0)$ & $13(14.4)$ \\
\hline Secondary & $13(43.3)$ & $10(33.3)$ & $14(46.7)$ & $37(41.1)$ & $9(30.0)$ & $10(33.3)$ & $9(30.0)$ & $28(31.1)$ \\
\hline $\begin{array}{l}\text { Tertiary } \\
\text { Monthly income }\end{array}$ & $14(46.7)$ & $19(63.3)$ & $12(40.0)$ & $45(50.0)$ & $15(50.0)$ & $19(63.3)$ & $15(50.0)$ & $49(54.4)$ \\
\hline$<\mathrm{RM} 3000$ & $15(50.0)$ & $12(40.0)$ & $15(50.0)$ & $42(46.6)$ & $15(50.0)$ & $6(20.0)$ & $14(46.7)$ & $35(38.9)$ \\
\hline $\begin{array}{l}\text { RM3000 } \\
- \text { RM6500 }\end{array}$ & $11(36.7)$ & $9(30.0)$ & $13(43.3)$ & $33(36.7)$ & $9(30.0)$ & $16(53.3)$ & $13(43.3)$ & $38(42.2)$ \\
\hline > RM6500 & $4(13.3)$ & $9(30.0)$ & $2(6.7)$ & $15(16.7)$ & $6(20.0)$ & $8(26.7)$ & $3(10.0)$ & $17(18.9)$ \\
\hline \multicolumn{9}{|l|}{ Medication } \\
\hline None & $17(56.7)$ & $29(96.7)$ & $14(46.7)$ & $60(66.7)$ & $9(30.0)$ & $16(53.3)$ & $8(26.7)$ & $33(36.7)$ \\
\hline \multicolumn{9}{|l|}{ Supplement } \\
\hline Yes & $3(10.0)$ & $8(26.7)$ & $2(6.7)$ & $13(14.4)$ & $5(16.7)$ & $5(16.7)$ & $3(10.0)$ & $13(14.4)$ \\
\hline No & $27(90.0)$ & $22(73.3)$ & $28(93.3)$ & $77(85.6)$ & $25(83.3)$ & $25(83.3)$ & $27(90.0)$ & $77(85.6)$ \\
\hline
\end{tabular}

Data are presented as mean \pm standard deviation or median (Interquartile range). 


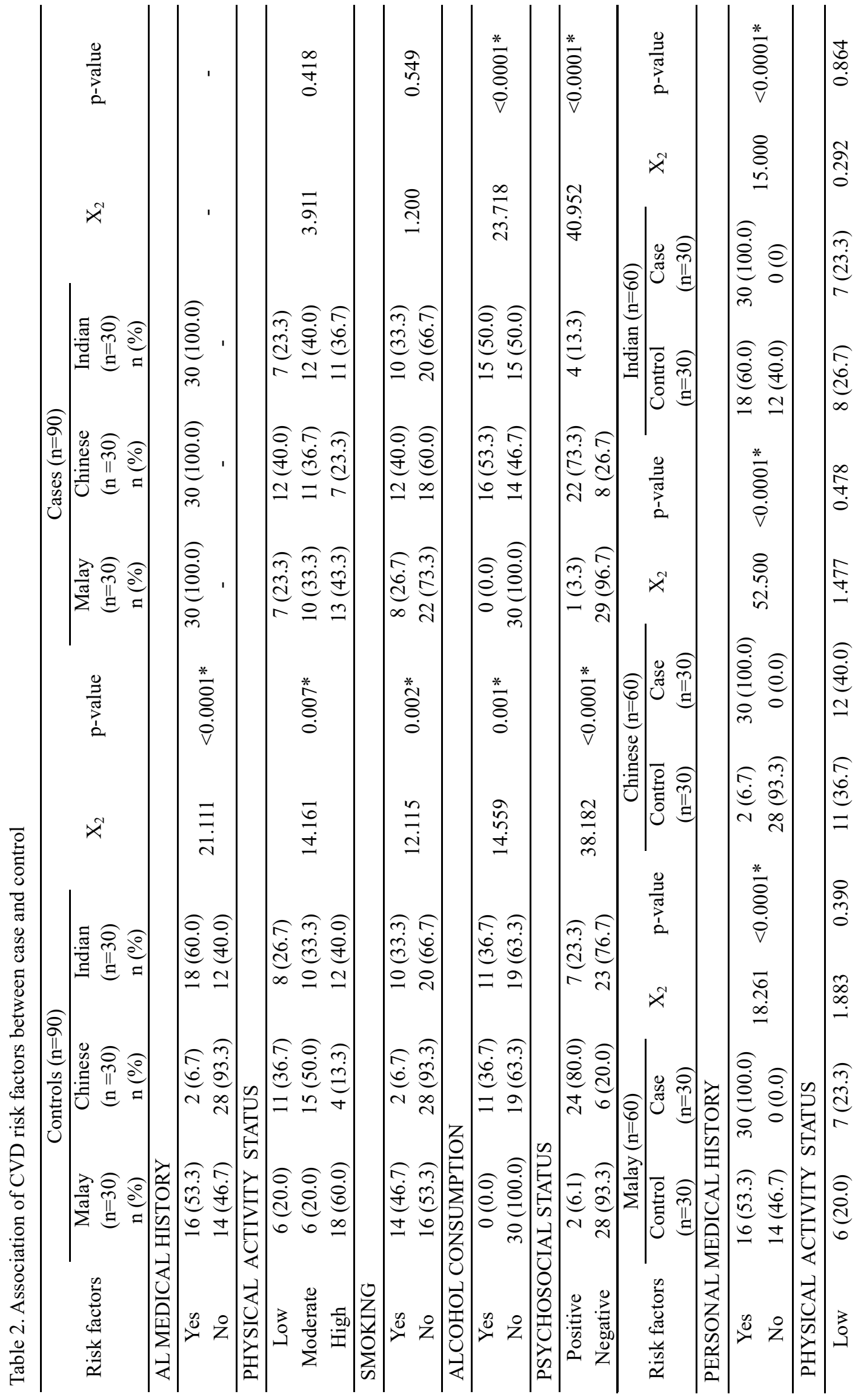




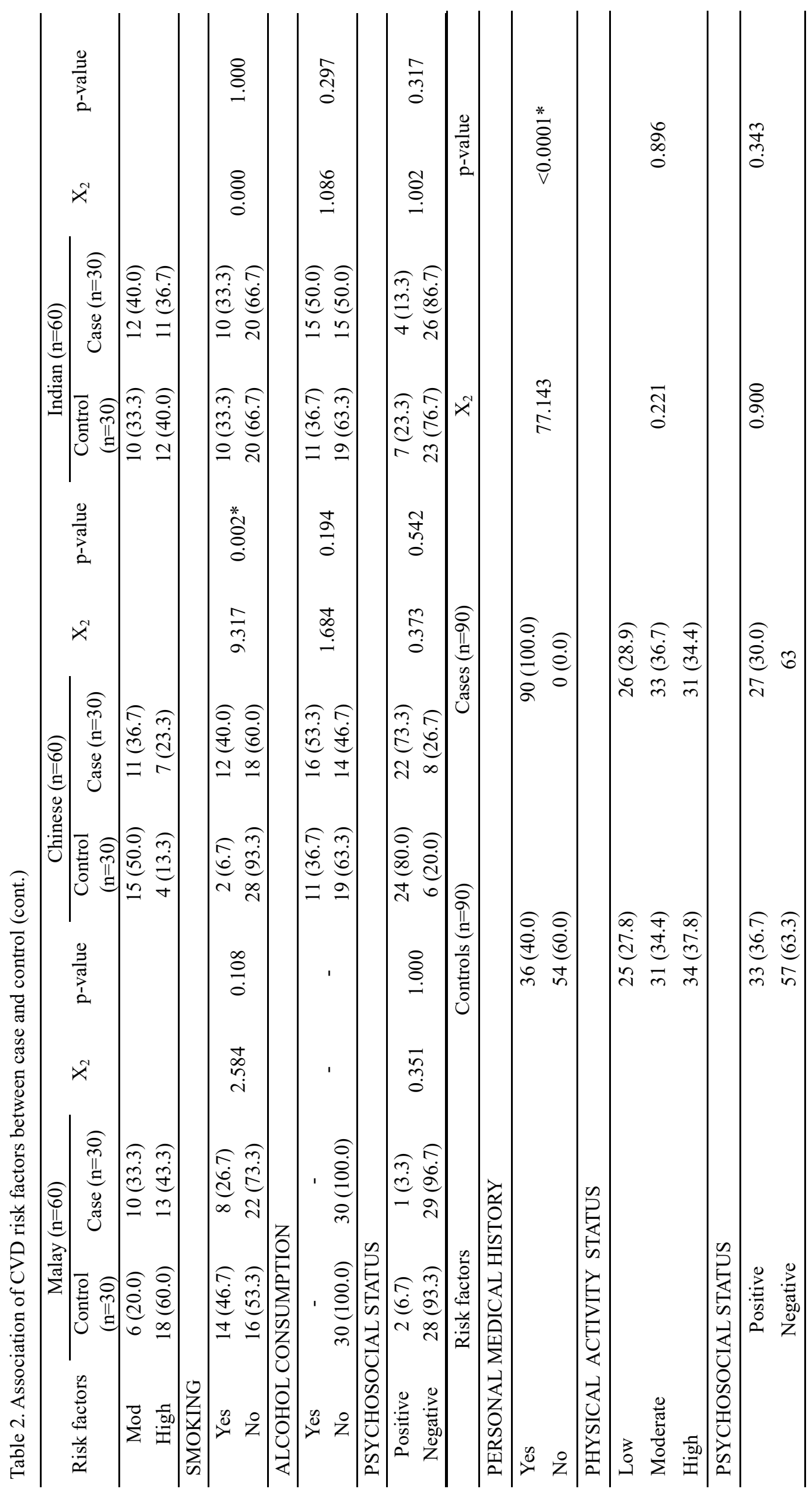




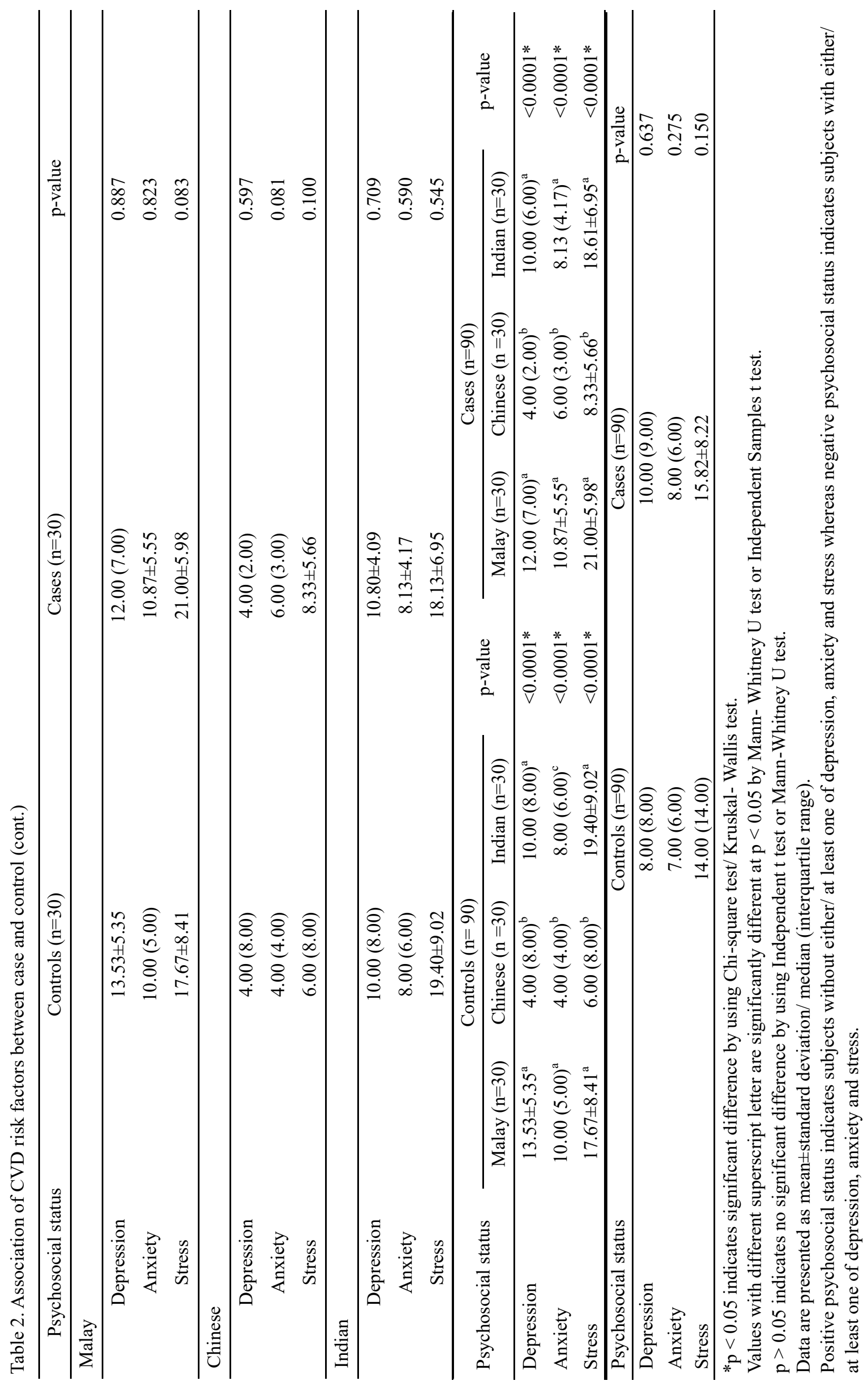


ஜ

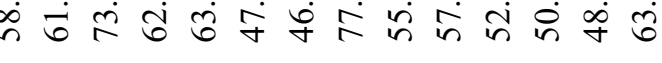

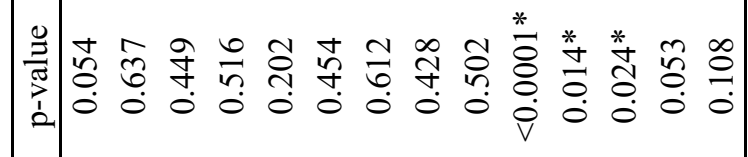

च으 m

in 0

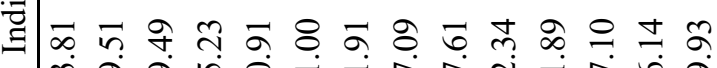

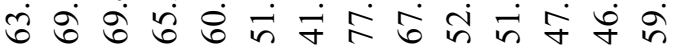

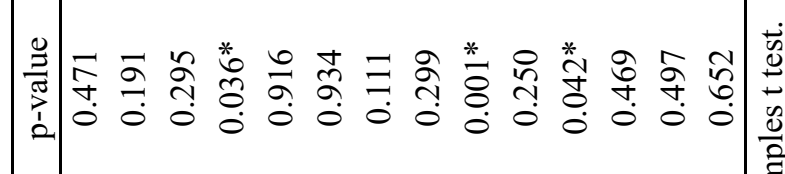

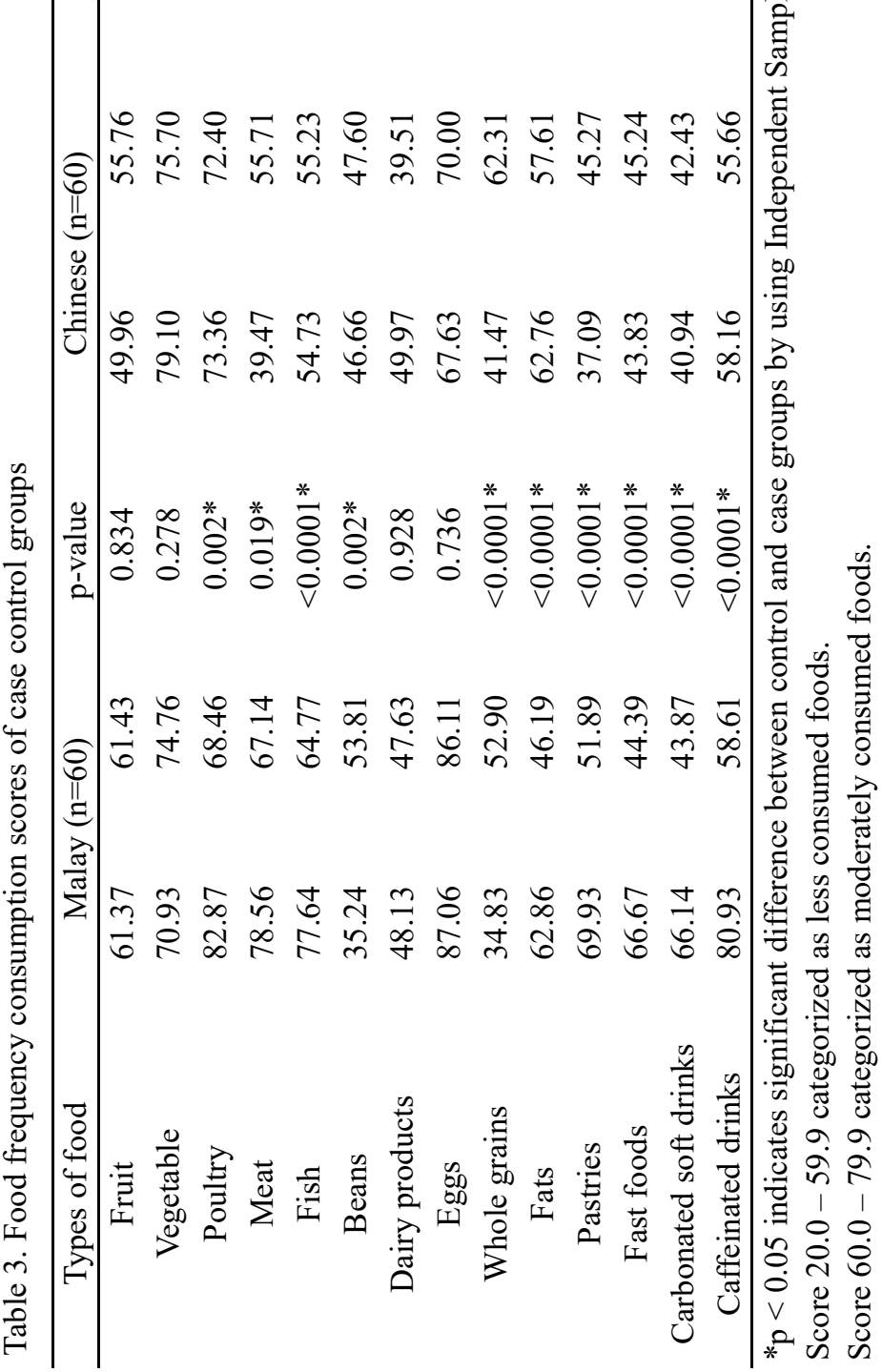




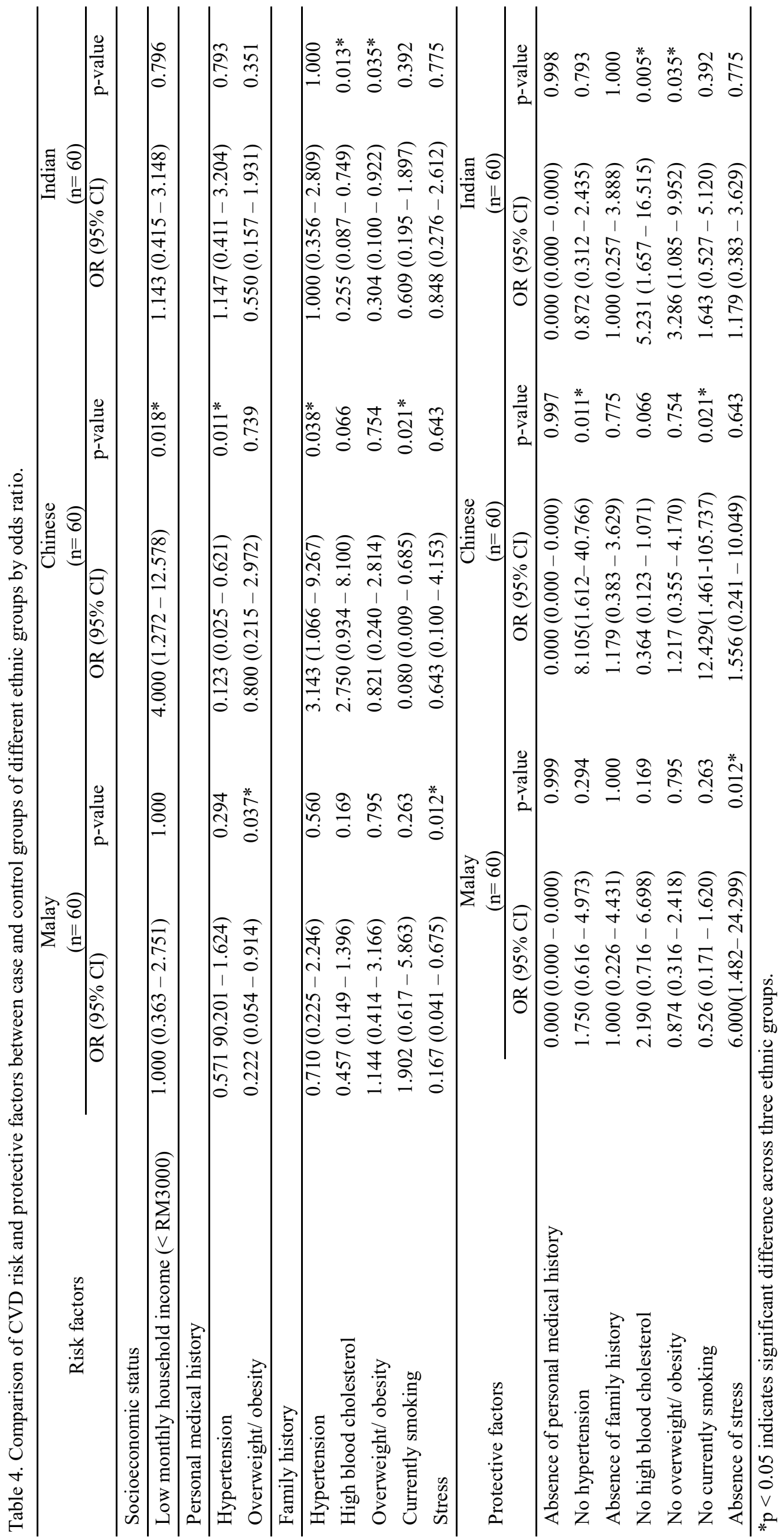




\subsection{Food frequency consumption of respondents}

Table 3 shows the food frequency consumption scores combining all the cases and controls according to ethnicity. All the controls and cases showed no significant differences in terms of the consumption of the 15 food items selected. By averaging the scores for both controls and cases groups, there was no food categorized under both extremely less and highly consumed foods, whereas fruit, nuts, beans, dairy products, whole grains, fats, pastries, fast foods and carbonated soft drinks were categorized as less consumed foods. While vegetable, poultry, meat, fish, eggs and caffeinated drinks were classified as moderately consumed foods. On the other hand, cases and controls exhibited great significant differences $(p<0.05)$ in terms of dietary pattern, and especially in terms of fats, protein, fast food, sugar and caffeinated drinks, according to ethnicity. For all ethnic groups, the consumption of pastries was statistically significant $(\mathrm{p}<0.05)$.

3.4 Comparison of risk and protective factors for cardiovascular disease between case and control groups by odds ratio

Table 4 shows the comparison of CVD risk and protective factors between case and control groups according to ethnicity by odds ratio (OR). For Malays, risk factors such as overweight/ obesity (OR: $0.222, \mathrm{CI}$ : $0.054-0.914)$ and stress (OR: 0.167, CI: $0.041-0.675)$ were statistically significant $(\mathrm{p}<0.05)$. Absence of stress with OR: 6.000 (CI: 1.482, 24.299) is the only factor which is statistically significant but not protective, with $\mathrm{p}=0.012$. For Chinese, risk factors such as low monthly household income (OR: 4.000, CI: 1.272-12.578), personal medical history of hypertension (OR: 0.123, CI: 0.025- 0.621), family history of hypertension (OR: 3.143, CI: $1.066-9.267$ ) and currently smoking (OR: 0.080, CI: $0.009-0.685$ ) were statistically significant (p $<0.05$ ). The inverse relationship between the absence of a family history of hypertension with the occurrence of CVD with OR less than 1 (OR: 0.318, CI: 0.108-0.938, p
$=0.038$ ), indicates it is statistically significant protective against CVD. Among Chinese, the factors with OR which were statistically significant but not protective against CVD due to large OR obtained included the absence of a personal medical history of hypertension (OR: 8.105, CI: 1.612-40.766) and not currently smoking (OR: 12.429, CI: 1.461-105.737). For Indians, only family history of high blood cholesterol (OR: 0.255 , CI: 0.087-0.749) and overweight/ obesity (OR: 0.304, CI: $0.100-0.922)$ were statistically significant with ( $\mathrm{p}<$ $0.05)$. Factors which were statistically significant but not protective included absence of a family history of high blood cholesterol (OR: 5.231, CI: 1.657-16.515) and no family history of overweight/ obesity (OR: 3.286, CI: $1.085-9.952$ ) with $\mathrm{p}=0.005$ and $\mathrm{p}=0.035$ respectively.

\subsection{Quality of life of respondents}

Table 5 shows the Physical Component Summaries (PCS) and Mental Component Summaries (MCS) of the respondents according to ethnicity. The scores of both PCS and MCS among Malay and Indian respondents, together with the PCS of Chinese respondents, are higher in controls than that of cases, in which only the PCS of Malay respondents showed significant difference $(p<$ $0.05)$ between groups. For MCS of Chinese, the scores are higher in cases than that in controls.

All controls and cases showed no significant differences $(p>0.05)$ across ethnicities. In the control group, the higher scores belong to Malays, followed Indian and Chinese for PCS while the highest scores for MCS belongs to Indian, Malay and lastly, Chinese. However, the difference in scores obtained for different ethnicity was not significant $(p>0.05)$. On the other hand, in the case group, the highest scores in PCS and MCS belongs to Indian, then followed by Chinese and Malay. All of these differences were not significant ( $p>$ 0.05). In addition, HRQoL scores of total controls were higher than that those of both PCS and MCS. However, only the difference in the scores for PCS showed significant difference $(\mathrm{p}<0.05)$. The PCS scores were

Table 5. Physical and mental component summary scores of multi ethnic respondents

\begin{tabular}{|c|c|c|c|c|}
\hline Quality of Life & \multicolumn{3}{|c|}{ Control $(\mathrm{n}=90)$} & $\mathrm{p}$-value \\
\hline Physical component summary (PCS) & $52.89(6.17)$ & $50.82 \pm 12.73$ & $51.64(10.03)$ & 0.268 \\
\hline Mental component summary (MCS) & $52.48(7.58)$ & $50.83(7.83)$ & $53.86(6.31)$ & 0.796 \\
\hline \multirow{2}{*}{ Quality of Life } & & Case $(n=90)$ & & \multirow{2}{*}{ p-value } \\
\hline & Malay $(n=30)$ & Chinese $(n=30)$ & $\operatorname{Indian}(\mathrm{n}=30)$ & \\
\hline Physical component summary (PCS) & $47.99(24.96)$ & $48.79(14.57)$ & $50.06(21.05)$ & 0.371 \\
\hline Mental component summary (MCS) & $51.00(10.08)$ & $53.56(4.31)$ & $53.58(4.47)$ & 0.080 \\
\hline Quality of Life & \multicolumn{2}{|c|}{ Control $(\mathrm{n}=90)$} & $\mathrm{n}=90)$ & p-value \\
\hline Physical component summary (PCS) & \multicolumn{2}{|c|}{$51.87(11.31)$} & 19.36) & $<0.0001^{*}$ \\
\hline Mental component summary (MCS) & \multicolumn{2}{|c|}{$53.24(8.59)$} & $(7.36)$ & 0.911 \\
\hline
\end{tabular}

${ }^{*} \mathrm{p}<0.05$ indicates significant difference by using Mann Whitney $\mathrm{U}$ test.

$p>0.05$ indicates no significant difference between ethnic groups by using Kruskal- Wallis test. 
relatively lower compared to MCS.

\section{Discussion}

CVD risk factors were significantly different according to ethnicity. Even in the same residential area, different ethnic groups possessed different eating patterns, SES and psychosocial status, contributing to different levels of significance for the CVD risk factors investigated.

Based on Table 2, controls without personal medical history contributed to lower CVD risk compared to cases. This was in line with the studies which showed that lower prevalence for personal medical history such as hypertension contributed to lower risk of in-hospital CVD mortality among respondents ( $\mathrm{Lu}$ and Nordin, 2013). In terms of physical activity, the finding obtained were in line with a study which revealed that when the physical activity involvement of population was investigated, the Chinese engaged significantly less physical activity (mean $=11.51, \mathrm{SD}=5.86)$ as compared to Malays $($ mean $=12.80, \mathrm{SD}=5.77)$ and other groups $($ mean $=15.14, \mathrm{SD}=5.22) \quad($ Lian et al., 2016). Differences in levels of physical activity may be due to the complex interaction between socio-economic, cultural, and physical environmental factors (Teh et al., 2011). Lu and Nordin (2013) also found that Malays had the highest prevalence of smoking in Malaysia as compared to other groups.

For monthly household income, Tan et al. (2011) discovered that Malays and other-Bumis tended to have lower household income than Indians, who tended to have lower household incomes than Chinese. For example, monthly household income for other-Bumis was roughly RM1200 per month, compared to RM1545 for Malays, RM2135 for Indians, and RM2535 for Chinese. This discovery was in line with the findings obtained. On the other hand, the Islamic background of Malay respondents which prohibits the consumption of alcohol contributes to a significant difference between ethnic groups. Malays occupied the highest prevalence of negative psychosocial status, as evidenced by a previous study which indicated that perceived stress was dominated by Malays $(52.2 \%)$, compared to all the other races (Jia and Loo, 2018). For both control and case groups, alcohol consumption and psychosocial status showed significant differences between ethnic groups. These risk factors were remarkable and should be further investigated in detail.

Overall, personal medical history showed significant differences between the controls and cases $(p<0.001)$, as greatly supported by a study which concluded that the prevalence of heart failure is higher in patients with personal diseases such as diabetes $(16 \%-31 \%)$ than normal subjects (4\% - 6\%) (Leon and Maddox, 2015). Malay and Indian respondents showed significant differences between groups $(p<0.001)$ in terms of the personal medical history of hypertension, diabetes mellitus, hypercholesterolemia, and stroke. On the other hand, Chinese respondents showed significant differences between case-control groups for personal medical history $(\mathrm{p}<0.001)$, in addition to smoking ( $\mathrm{p}=$ $0.002)$. This was evidenced via a prospective cohort study which showed the hazard ratios (HR) for all-cause CVD were 1.7 for current smokers, 1.07 for former smokers, and 1 for never smokers (McEvoy et al., 2015).

The scores for psychosocial status were the highest in Malays, followed by Indians and Chinese. The highest score for all the three components of psychosocial status is stress, followed by depression and lastly anxiety which was in line with a study conducted by Saadat et al. (2015). This finding is inconsistent with a study which reported a significant relationship $(p<0.05)$ between the case and control for the psychosocial status, in which cases with higher scores for psychosocial status was then identified as at increased risk for incident CHD and health behaviors (Cohen et al., 2018).

Almost all food groups were dependent on ethnicity, but not lipid profile status. Poultry and meat intake has been positively associated with the increase risk of CVD through several studies (Micha et al., 2010; Pan et al., 2012). For specific CVD mortality, red meat intake was positively associated with ischemic heart disease when women and men were combined $(\mathrm{HR}=1.41,95 \% \mathrm{CI}=$ 1.05-1.89, p-value $=0.04)$ (Takata et al., 2013). Additionally, Mozaffarian and Rimm (2006) reported that modest consumption of fish (1 to 2 servings per week), especially those higher in the omega-3 fatty acids, reduced the risk of coronary death by $36 \%(95 \% \mathrm{CI}$ : $20 \%$ to $50 \%, \mathrm{p}<0.001)$ and total mortality by $17 \%(95 \%$ CI: $0 \%$ to $32 \%, p=0.046)$. A study showed Malays possessed highest fish intake as compared to other ethnics. This finding was in line with the study that Malaysians consumed $168 \mathrm{~g}$ /day fish, with Malays' consumption of fish $(175 \pm 143 \mathrm{~g} /$ day $)$ significantly $(\mathrm{p}<$ $0.001)$ higher compared with the other two ethnic groups $($ Chinese $=152 \pm 133 \mathrm{~g} /$ day, Indians $=136 \pm 141 \mathrm{~g} /$ day $)$ (Ahmad et al., 2016).

Next, the consumption of nuts, beans and wholegrain were dependent on ethnicity, and not dependent on groups. Fats, especially 'bad fats' such as trans fats and saturated fats in various foods, are considered the main contributor to CVD from foods (Briggs et al., 2017). The 2013 American Heart Association and American College of Cardiology (AHA/ACC) Guideline on Lifestyle 
Management to Reduce Cardiovascular Risk reports strong evidence (level A) for reducing SFA intake (5\% to $6 \%$ of calories) to lower LDL cholesterol (Eckel et al., 2014). High intake of Western-style food with high saturated and trans fats, sodium and sugar contribute to various health impacts. Western-style food intake varies according to ethnicity, particularly due to different occupations, education levels and family background (Osei-Kwasi et al., 2016). The present study showed that Malays consumed more fats, fast food and sugary drinks, followed by Indian and Chinese. This finding was in line with a previous study that Malay had significantly higher scores for the Western-based food pattern as compared to Chinese (Abdullah et al., 2016).

The previous study demonstrates that for the general population of healthy adults, moderate caffeine consumption of $400 \mathrm{mg} / \mathrm{d}$ equivalent to 4 cups of brewed coffee is not associated with toxicity, cardiovascular effects, effects on bone status and calcium balance (with consumption of adequate calcium) (Turnbull et al., 2017). Basically, there was no significant difference in terms of caffeine consumption except for Malay respondents in which its controls had higher intake than that of cases. Thus, it can be concluded that caffeine consumption was not associated with CVD risk.

There are contradictory findings, as the consumption of poultry, meat, fats, pastries, fast foods, carbonated soft drinks, and caffeinated were higher in the control group as compared to case groups. In addition to the higher consumption of fish, nuts, beans and whole grains in the case group, this can be best explained by increased educational level and awareness of CVD among cases. This allows them to be aware and more concern about the foods which they will consume in order to reduce the risk for CVD. This leads to the development and implementation of action plans against CVD. Thus, taking corrective action should include consuming the right foods to fight against health complications.

Based on Table 4, factors such as overweight/obesity and stress for Malays in addition to the family history of high blood cholesterol and overweight/obesity for Indians were negatively associated $(\mathrm{OR}<1)$ with CVD. This finding can be best explained by the continuously increasing in the health and nutrition awareness among overweight/ obese people, especially towards CVD risk. They tend to develop and implement an efficient action plan targeting CVD prevention in order to avoid further weight gain. In addition, suitable normal stress allows people to fit their motivational orientation and, thus, lead to a regulatory fit. In the working world, this pursuit could lead to attuning the regulatory focus of the workers to the working environment in order to achieve better performance.

On the other hand, low-income level and family history of hypertension were positively associated (OR > 1) with CVD among Chinese. This can be best explained by low-income families not being able to afford more expensive healthy food, and thus spending their limited resources on 'cheaper' food sources such as fast food and sweetened beverages. The contradictory findings for Chinese obtained from this study were personal medical history of hypertension (OR: 0.123, CI: 0.025-0.621) and currently smoking (OR: 0.080, CI: 0.009-0.685). This can be explained by the reason for higher education and increase awareness towards the prevention of CVD among the hypertensive population and smokers, thus protecting them from CVD risk. Among all ethnic groups, Chinese had the highest number of significant risk factors for CVD.

The significant protective factor for Chinese is the absence of the family history of hypertension with the occurrence of CVD with OR less than 1. A total of 2,302 male and female Framingham offspring study participants with parental history of premature CVD (father less than 55 years and mother less than 65 years) were diagnosed for CVD risk. CVD increased $75 \%$ with paternal and about $60 \%$ with maternal history of premature CVD after 8 years of follow-up (Kolber and Scrimshaw, 2014).

Absence of personal medical history of hypertension and not currently smoking were statistically significant but not protective against CVD due to the large OR obtained among Chinese population. For Malay, absence of stress is the only factor which is statistically significant with $p=0.012$. However, it is not protective factor as the OR was much greater than 1. For Indian, factors which were statistically significant but not protective were the absence of the family history of high blood cholesterol and no family history of overweight/ obesity. These findings can be best explained for several reasons. Although respondents were not under hypertension condition, there are still many other confounding factors such as unhealthy diet, physical inactivity and alcohol consumption might influence the risk for CVD. Besides, this may be due to the limitation that all respondents involved were all located in Dengkil, a small town in Selangor, which may not have been comprehensive enough.

Besides, the finding obtained which showed there was no significant difference $(p<0.05)$ in HRQoL scores according to ethnicity also in line with the findings obtained via a cross-sectional study involving 652 of multiethnic adolescents (aged 13 years) which showed the slight difference in the HRQoL scores were 
not significant ( $\mathrm{p}>0.05)$ (Loh et al., 2015). According to Loh et al. (2015), Chinese occupied the highest scores (mean: 71.15, CI: 65.28, 77.02), followed by Indians (mean: 69.91, CI: 64.23, 75.60) and lastly, Malays (mean: 69.53, CI: 68.12, 70.93). However, the prevalence of HRQoL scores in the present study was not consistent among the ethnic groups most probably due to sociodemographic resources, clinical and environmental factors (Leow et al., 2013).

The study also found that HRQoL scores of total controls were higher than that of cases for both the PCS and MCS. This shows that the control group had higher quality of life compared to the case group in terms of physical and mental health. However, only the difference in the scores for PCS showed significant difference $(\mathrm{p}<$ 0.05). The PCS score was relatively lower compared to MCS. The mean scores of PCS and MCS among 596 Malaysian adults with mean age of 35.7 years were 48.9 and 49.1, respectively (Shafie et al., 2011). Therefore, PCS and MCS scores of current respondents were considered normal as they were slightly higher than the average scores of the Malaysian population.

\section{Conclusion}

It could be concluded that generally, all the risk factors were independent on the total cholesterol status except for personal medical history. Chinese is the ethnic group with the highest CVD risk as due to the highest number of significant CVD risk factors. With the determination of each factor in each ethnicity, strategies of nutrition management of CVD can be identified easily. Further study might better consider research on randomly selected samples with the intention to increase the validity of the research. Besides, FFQ should be improved by including the type of food and drinks consumed, food preparation methods, and portion size. Anthropometric measurements and blood withdrawals should be implemented to obtain the actual serum lipid profile and accurate body weight and height.

\section{Acknowledgement}

The authors would like to express their gratitude to all the adult respondents for their participation, full cooperation, and patience with the study. Also, highest appreciations go to those who assisted in completing this project, including Mr Liew Rock Keey.

\section{References}

Abdullah, N-.F., Teo, P.S. and Foo, L.H. (2016). Ethnic differences in the food intake patterns and its associated factors of adolescents in Kelantan, Malaysia. Nutrients, 8(9), 551. https:// doi.org/10.3390/nu8090551

Ahmad, N.I., Wan Mahiyuddin, W.R., Tengku Mohamad, T.R., Ling, C.Y., Daud, S.F., Hussein, N.C., Abdullah, N.A., Shaharudin, R. and Sulaiman, L.H. (2016). Fish consumption pattern among adults of different ethnics in Peninsular Malaysia. Food and Nutrition Research, 60(1), 1-15. https:// doi.org/10.3402/fnr.v60.32697

Akter, S.F.U., Fauzi, A., Nordin, M., Satwi, S., Mohamed, A., Aznan, M. and Samsul, D. (2010). Prevalence of cardiovascular risk factors in a selected community at Kuantan, Pahang, Malaysia. International Journal of Medicine and Medical Sciences, 2, 322-328.

Alvi, M. (2016). A manual for selecting sampling techniques in research. Munich Personal RePEc Archive, p. 1-57. Pakistan: University of Karachi.

André, N., Klopp-Dutote, N., Biet-Hornstein, A., Strunski, V. and Page, C. (2018). Cardiovascular risk and severity factors in patients admitted to hospital for spontaneous epistaxis. Eur. Annals of Otorhinolaryngology, Head and Neck Diseases, 135 (2), 119-122. https://doi.org/10.1016/ j.anorl.2017.11.004

Aniza, I., Nurmawati, A., Hanizah, Y. and Ahmad Taufik, J. (2016). Modifiable risk factors of cardiovascular disease among adults in rural community of Malaysia: A cross sectional study. Malaysian Journal of Public Health Medicine, 16, 53-61.

Briggs, M.A., Petersen, K.S. and Kris-Etherton, P.M. (2017). Saturated fatty acids and cardiovascular disease: Replacements for saturated fat to reduce cardiovascular risk. Healthcare. Multidisciplinary Digital Publishing Institute, 5(2), 29. https:// doi.org/10.3390/healthcare5020029

Department of Statistics. (2015). Dengkil. Retrieved on March 26, 2018 from Department of Statistics website: https://www.citypopulation.de/php/ malaysia-mukim-admin.php?adm2id=100701

Department of Statistics. (2017). Statistics on causes of death, Malaysia. Retrieved on March 26, 2018 from Department of Statistics website: https:// www.dosm.gov.my/v1/index.php?r=column/ pdfPrev\&id=Y3psYUI2VjU0ZzRhZU1kcVFMMTh GUT09

Eckel, R.H., Jakicic, J.M., Ard, J.D., De Jesus, J.M., Miller, N.H., Hubbard, V.S., Lee, I.-M., Lichtenstein, A.H., Loria, C.M. and Millen, B.E. (2014). 2013 AHA/ACC guideline on lifestyle management to reduce cardiovascular risk: A report of the American College of Cardiology/American 
Heart Association task force on practice guidelines. Journal of American College of Cardiology, 63(25, Part B), 2960-2984. https://doi.org/10.1016/ j.jacc.2013.11.003

Gandek, B., Sinclair, S.J., Kosinski, M. and Ware Jr, J.E. (2004). Psychometric evaluation of the sf-36 ${ }^{\circledR}$ health survey in medicare managed care. Health Care Financing Review, 25(4), 5-25.

Greenland, S., Senn, S.J., Rothman, K.J., Carlin, J.B., Poole, C., Goodman, S.N. and Altman, D.G. (2016). Statistical tests, p-values, confidence intervals, and power: A guide to misinterpretations. European Journal of Epidemiology, 31, 337-350. https:// doi.org/10.1007/s10654-016-0149-3

IPAQ Analysis. (2005). Guidelines for Data Processing and Analysis of the International Physical Activity Questionnaire (IPAQ). Retrieved on March 26, 2018 from IPAQ Analysis website: https:// www.researchgate.net/file.PostFileLoader.html? $\mathrm{id}=5641 \mathrm{f} 4 \mathrm{c} 36143250 \mathrm{eac} 8 \mathrm{~b} 45 \mathrm{~b} 7 \&$ assetKey $=\mathrm{AS} \%$ 3A294237418606593\%401447163075131

Jia, Y. and Loo, Y. (2018). Prevalence and determinants of perceived stress among undergraduate students in a Malaysian university. Journal of Health and Translational Medicine, 21(1), 1-4.

Juhan, N., Zubairi, Y.Z., Zuhdi, A., Khalid, Z.M. and Ahmad, W.A.W. (2018). Gender differences in mortality amongst elevation myocardial infarction patients in Malaysia from 2006 to 2013. Annals of Saudi Medicine, 38(1), 1-7. https:// doi.org/10.5144/0256-4947.2018.1

Kelsey, J.L., Whittemore, A.S., Evans, A.S. and Thompson, W.D. (1996). Methods in Observational Epidemiology, Monographs in Epidemiology and Biostatistics, p. 1 - 444. New York: Oxford University Press Inc.

Kolber, M.R. and Scrimshaw, C. (2014). Family history of cardiovascular disease. Canadian Family Physician, 60, 1016.

Kumar, A. (2014). Changing trends of cardiovascular risk factors among Indians: A review of emerging risks. Asian Pacific Journal of Tropical Biomedicine, 4(12), 1001-1008. https://doi.org/10.12980/ APJTB.4.201414B401

Leon, B.M. and Maddox, T.M. (2015). Diabetes and cardiovascular disease: Epidemiology, biological mechanisms, treatment recommendations and future research. World Journal of Diabetes, 6(13), 12461258. https://doi.org/10.4239/wjd.v6.i13.1246

Leow, M.K.-S., Griva, K., Choo, R., Wee, H.-L., Thumboo, J., Tai, E.S. and Newman, S. (2013). Determinants of health-related quality of life
(HRQoL) in the multiethnic Singapore population-a national cohort study. PloS One, 8, 67-138. https:// doi.org/10.1371/journal.pone.0067138

Lian, T.C., Bonn, G., Han, Y.S., Choo, Y.C. and Piau, W.C. (2016). Physical activity and its correlates among adults in Malaysia: A cross-sectional descriptive study. PloS One, 11, 73-157. https:// doi.org/10.1371/journal.pone.0157730

Lim, K.S., Ng, C.C., Chan, C.K., Foo, W.S., Low, J.S.Y. and Tan, C.T. (2017). Ethnic variation of genetic (idiopathic) generalized epilepsy in Malaysia. Seizure, 45, 24-27. https://doi.org/10.1016/ j.seizure.2016.11.011

Loh, D., Moy, F., Zaharan, N. and Mohamed, Z. (2015). Disparities in health-related quality of life among healthy adolescents in a developing country-the impact of gender, ethnicity, socio-economic status and weight status. Child Care Health and Development, 41(6), 1216-1226. https:// doi.org/10.1111/cch.12252

Lovibond, P.F. and Lovibond, S.H. (1995). The structure of negative emotional states: Comparison of the Depression Anxiety Stress Scales (DASS) with the beck depression and anxiety inventories. Behaviour Research and Therapy, 33(3), 335-343. https:// doi.org/10.1016/0005-7967(94)00075-U

Lu, H.T. and Nordin, R.B. (2013). Ethnic differences in the occurrence of acute coronary syndrome: Results of the Malaysian National Cardiovascular Disease (NCVD) database registry (March 2006-February 2010). BMC Cardiovascular Disorders, 13, 97. https://doi.org/10.1186/1471-2261-13-97

Mathieson, K. (2014). Making sense of biostatistics: Types of probability sampling. Journal of Clinical Research Best Practices, 10(10), 192-245.

McEvoy, J.W., Blaha, M.J., Defilippis, A.P., Lima, J.A., Bluemke, D.A., Hundley, W.G., Min, J.K., Shaw, L.J., Lloyd-Jones, D.M. and Barr, R.G. (2015). Cigarette smoking and cardiovascular events: Role of inflammation and subclinical atherosclerosis: The multiethnic study of atherosclerosis. Arteriosclerosis, Thrombosis, and Vascular Biology, 35, 700-709. https://doi.org/10.1161/ ATVBAHA.114.304562

Micha, R., Michas, G. and Mozaffarian, D. (2012). Unprocessed red and processed meats and risk of coronary artery disease and type 2 diabetes-an updated review of the evidence. Current Atherosclerosis Reports, 14, 515-524. https:// doi.org/10.1007/s11883-012-0282-8

Mozaffarian, D. (2016). Dietary and policy priorities for cardiovascular disease, diabetes, and obesity: A 
comprehensive review. Circulation, 133, 187-225. https://doi.org/10.1161/

\section{CIRCULATIONAHA.115.018585}

Osei-Kwasi, H.A., Nicolaou, M., Powell, K., Terragni, L., Maes, L., Stronks, K., Lien, N. and Holdsworth, M. (2016). Systematic mapping review of the factors influencing dietary behaviour in ethnic minority groups living in Europe: A dedipac study. Internationa Journal of Behavioural Nutrition and Physical Activity, 13, 85. https://doi.org/10.1186/ s12966-016-0412-8

Pan, A., Sun, Q., Bernstein, A.M., Schulze, M.B., Manson, J.E., Stampfer, M.J., Willett, W.C. and Hu, F.B. (2012). Red meat consumption and mortality: Results from 2 prospective cohort studies. Archives of Internal Medicine, 172, 555-563. https:// doi.org/10.1001/archinternmed.2011.2287

Pandey, A.K., Pandey, S., Blaha, M.J., Agatston, A., Feldman, T., Ozner, M., Santos, R.D., Budoff, M.J., Blumenthal, R.S. and Nasir, K. (2013). Family history of coronary heart disease and markers of subclinical cardiovascular disease: Where do we stand? Atherosclerosis, 228(2), 285-294. https:// doi.org/10.1016/j.atherosclerosis.2013.02.016

Roth, G.A., Huffman, M.D., Moran, A.E., Feigin, V., Mensah, G.A., Naghavi, M. and Murray, C.J. (2015). Global and regional patterns in cardiovascular mortality from 1990 to 2013. Circulation, 132, 16671678. https://doi.org/10.1161/ CIRCULATIONAHA.114.008720

Saadat, M., Behboodi, Z.M. and Saadat, E. (2015). Comparison of depression, anxiety, stress, and related factors among women and men with human immunodeficiency virus infection. Journal of Human Reproductive Sciences, 8(1), 48-51. https:// doi.org/10.4103/0974-1208.153128

Schmidt, C.O. and Kohlmann, T. (2008). When to use the odds ratio or the relative risk? International Journal of Public Health, 53, 165-167. https:// doi.org/10.1007/s00038-008-7068-3

Shafie, A.A., Hassali, M.A. and Liau, S.Y. (2011). A cross-sectional validation study of EQ-5D among the Malaysian adult population. Quality of Life Research, 20(4), 593-600. https://doi.org/10.1007/ s11136-010-9774-6

Song, J.W. and Chung, K.C. (2010). Observational studies: Cohort and case-control studies. Plastic and Reconstructive Surgery, 126(6), 2234-2242. https:// doi.org/10.1097/PRS.0b013e3181f44abc

Takata, Y., Shu, X.-O., Gao, Y.-T., Li, H., Zhang, X., Gao, J., Cai, H., Yang, G., Xiang, Y.-B. and Zheng, W. (2013). Red meat and poultry intakes and risk of total and cause-specific mortality: Results from cohort studies of Chinese adults in Shanghai. PloS One, 8, 59-63. https://doi.org/10.1371/ journal.pone.0056963

Teh, P.L., Yong, C.C., Chong, C.W. and Yew, S.Y. (2011). Do the Big Five Personality Factors affect knowledge sharing behaviour? A study of Malaysian universities. Malaysian Journal of Library and Information Science, 16(1), 47-62

Thompson, D.R., Ski, C.F., Garside, J. and Astin, F. (2016). A review of health-related quality of life patient-reported outcome measures in cardiovascular nursing. European Journal of Cardiovascular Nursing, $\quad 15(2), \quad 114-125 . \quad$ https:// doi.org/10.1177/1474515116637980

Thon, C.C., Yein, L.P. and Lian, C.W. (2012). The prevalence of cardiovascular risk factors in the young and middle-aged rural population in Sarawak, Malaysia. Malaysian Journal of Medical Science, 19 (2), 27-34.

Turnbull, D., Rodricks, J.V., Mariano, G.F. and Chowdhury, F. (2017). Caffeine and cardiovascular health. Regulatory Toxicology and Pharmacology, 89, 165-185. https://doi.org/10.1016/ j.yrtph.2017.07.025

Ware, J.E., Keller, S.D. and Kosinski, M. (1994). Sf-36 physical and mental health summary scales. Health Assessment Lab, p. 30-56. Boston, USA: New England Medical Center. 